\title{
FORMAÇÃO DE PROFESSORES: UMA ARQUITETURA PEDAGÓGICA COM FOCO NA M-LEARNING
}

\author{
Anna Helena Silveira Sonego - PPGEDU/UFRGS - sonego.anna@ gmail.com \\ Ana Carolina Ribeiro Ribeiro - PPGEDU/UFRGS - carolribeiro2@ gmail.com \\ Leticia Rocha Machado - PPGEDU/UFRGS - leticiarmachado@ yahoo.com.br \\ Patricia Alejandra Behar - PPGIE/PPGEDU/UFRGS - pbehar@terra.com.br
}

Resumo: Este artigo apresenta o desenvolvimento de uma Arquitetura Pedagógica (AP) para a M-Learning com foco na formação de professores. Para tanto, foi utilizado o objeto de aprendizagem EduMobile que apresenta diversos tipos de materiais digitais e visa introduzir estratégias para aplicação da $M$-Learning em contexto escolar. A referida AP foi aplicada em um curso de formação de professores realizado na Universidade Federal do Rio Grande do Sul. A metodologia do trabalho foi de abordagem qualitativa do tipo estudo de caso. Os dados foram coletados de duas formas: a) através das participações das atividades no Ambiente Virtual de Aprendizagem (AVA) utilizado; b) por meio de questionário aplicado no final das atividades. A análise dos dados apontou que uma AP para M-Learning precisa contemplar aplicativos, conteúdos interativos para dispositivos móveis (DM), objetivos com foco nos DM e metodologias que possibilitem a interatividade, mobilidade e conexão com a internet. Neste sentido, entende-se que o professor que pretende fazer uso destes aparelhos pode utilizar esta AP para guiá-lo e orientá-lo nesse processo.

Palavras-chave: formação de professores; m-learning; arquitetura pedagógica.

\section{TEACHER TRAINING: A PEDAGOGICAL ARCHITECTURE FOCUSED ON M-LEARNING}

\begin{abstract}
This article presents the development of a Pedagogical Architecture (PA) for $M$-Learning with a focus on teacher education. For this purpose, the learning object EduMobile was used to present several types of digital materials and aims to introduce strategies for applying M-Learning in a school context. In this sense, said AP was applied in a teacher training course at Federal University of Rio Grande do Sul. The methodology of the study was a qualitative approach of the case study type. The data were collected in two ways: a) through the participation of activities in the Virtual Learning Environment (VLE) used; b) questionnaire applied at the end. The analysis of the data pointed out that an AP for M-Learning is necessary to contemplate applications, interactive contents for mobile devices (MDs), objectives with focus on the MDs and methodologies that allow interactivity, mobility and connection to the internet. In this sense, the teacher who intends to use these devices can use this AP to guide and guide him in this process.
\end{abstract}

Keywords: teacher training; m-learning; pedagogical architecture

\section{INTRODUÇÃO}

A utilização de smartphones e tablets é uma realidade cada vez mais presente no cotidiano. Observa-se que a cada ano cresce o número de dispositivos móveis comercializados, possibilitando que diferentes faixas-etárias tenham acesso a estes aparelhos. Atualmente, os jovens representam 83\% (CETIC, 2016) do público que manuseia estas tecnologias. Portanto, percebe-se a importância de também incluí-las nos espaços escolares. 
As tecnologias podem ser cada vez mais inseridas nos ambientes educacionais. No entanto, é pertinente conciliá-los ao uso pedagógico, sendo necessária uma formação inicial e continuada dos professores de modo que possam incluir os dispositivos móveis em suas ações docentes. Uma possibilidade é através da M-Learning, ou em português: aprendizagem móvel. Segundo a UNESCO (2014, p.7), essa aprendizagem “[...] envolve o uso de tecnologias móveis, isoladamente ou em combinação com outras Tecnologias de Informação e Comunicação (TIC), a fim de permitir a aprendizagem a qualquer hora e em qualquer lugar".

Existem poucas iniciativas que buscam mapear ações que possam incluir os dispositivos móveis como possíveis práticas inclusivas nas instituições de ensino, seja em escolas, sejam em universidades. Assim, entende-se que o professor pode desenvolver na sua prática a aplicação de uma Arquitetura Pedagógica (AP), que pode ser estruturada através de diferentes elementos. As definições de AP são distintas. Neste contexto é utilizada a de Behar (2009, p.24) que a define como sendo: um sistema de premissas teóricas "que representa, explica e orienta a forma como se aborda o currículo e que se concretiza nas práticas pedagógicas e nas interações professor-aluno-objeto de estudo/conhecimento". Para a autora, a elaboração de uma AP deve contemplar os aspectos organizacionais, de conteúdo, metodológicos e tecnológicos com elementos que possibilitem a sua aplicação. Dessa forma, acredita-se na capacidade de reformulação das práticas pedagógicas, que podem gerar inovações didático-metodológicas, principalmente se considerado o uso de Objetos de Aprendizagem (OA). Um OA pode ser compreendido como qualquer material ou recurso digital que possua uma finalidade educacional e embasamento pedagógico (Behar et al, 2009a), podendo ser citados como exemplos vídeos, imagens, mapas, apresentações, páginas Web, entre outras. Os objetos podem ser utilizados como módulos de um determinado conteúdo ou possuir uma estrutura completa, sendo mais abrangente. Compreende-se que é essencial, assim, promover ambientes de formação continuada de modo a proporcionar espaços de reflexão e de aprimoramento da prática pedagógica.

O presente artigo tem por objetivo propor e analisar uma Arquitetura Pedagógica para a M-Learning com foco na formação de professores. Além dos elementos de cada aspecto da AP (tecnológico, metodológico e organizacional), foi utilizado como conteúdo, o Objeto de Aprendizagem EduMobile: Estratégias pedagógicas para o uso da M-Learning em sala de aula. Este tem como objetivo auxiliar o professor de diferentes níveis de ensino no desenvolvimento de estratégias pedagógicas que visem a utilização das tecnologias móveis, resinificando essas ferramentas e proporcionando o uso voltado para a aprendizagem das mesmas. Entende-se que o desenvolvimento desta AP pode auxiliar o professor a utilizar pedagogicamente os dispositivos móveis, já que propicia subsídios para uma reflexão sobre a temática e sua aplicação nas escolas.

A fim de contemplar os assuntos propostos a organização do presente estudo apresenta seis seções. Na segunda é abordado o conceito de Arquitetura Pedagógica e sua relação com a M-Learning. Na terceira seção destaca-se o uso de objeto de aprendizagem na formação de professores. Na quarta seção, é apresentada a metodologia adotada neste estudo. Na quinta seção, os resultados obtidos e, por fim, na sexta e última seção, as considerações finais.

\section{ARQUITETURA PEDAGÓGICA: UM FOCO NA M-LEARNING}

As Arquiteturas Pedagógicas (AP) são utilizadas para a organização de múltiplos espaços educacionais, em diferentes áreas do conhecimento. $\mathrm{O}$ conceito utilizado neste artigo é o de Behar (2009) que salienta que uma AP vai além da orientação e explicação 
dos elementos curriculares, apresentando quatro aspectos que a compõem, sendo eles:

- Aspectos organizacionais: relacionados ao planejamento, organização do tempo e espaço, expectativas e propostas pedagógicas, objetivos, atores, público-alvo e modalidade de ensino;

- Aspecto de conteúdo: relacionado aos materiais didáticos (vídeos, áudios, imagens, objetos de aprendizagem, entre outros), recursos e atividades de estudo;

- Aspectos metodológicos: formas de comunicação, interação e procedimentos de avaliação;

- Aspectos tecnológicos: escolha de um ambiente virtual de aprendizagem e de outras tecnologias.

Pensar nesses aspectos é essencial tendo em vista que, infelizmente, o uso de dispositivos móveis ainda não está associado à necessidade de um planejamento, acarretando em um uso limitado, já que não é possível usufruir todo o seu potencial. Portanto, entende-se que a organização de uma AP com foco na M-Learning pode oferecer condições para uma reflexão de como utilizar os dispositivos móveis mais contextualizados (metarreflexão) e com foco na construção do conhecimento. Dessa forma, é indispensável realizar um planejamento considerando o público-alvo, os conteúdos, as tecnologias, a metodologia, a modalidade, e tempo e contexto em que estes serões desenvolvidos.

Nesta perspectiva, compreende-se que a M-Learning possibilita ampliar as formas de leitura, escrita, pesquisa e construção de conteúdo potencializando o processo de ensino e de aprendizagem dos estudantes. Acredita-se que uma AP com foco na $M$ Learning pode gerar condições tanto para o professor quanto para o estudante de "usar aparelhos móveis para acessar recursos educacionais, conectar-se a outras pessoas, criar conteúdos, dentro ou fora da sala de aula" (UNESCO, 2014, p. 7). Sendo assim, a proposta desta AP serve como uma possibilidade de ensinar e aprender a partir da M-Learning, bem como, a compreensão da necessidade de reformulação nas atividades escolares a fim de gerar inovação didática e metodológica nas ações docentes, associada a integração do uso dos dispositivos móveis. Para que haja esta integração é pertinente utilizar materiais, como os objetos de aprendizagem, que trazem em sua essência situações problemas sobre a realidade das escolas, gerando reflexões e análises sobre o tema. Portanto, entende-se que OA podem auxiliar os professores no seu processo de formação e de ação docente para utilizar a tecnologia móvel e elaborar novas estratégias pedagógicas que possibilitem a formação do sujeito no âmbito educacional, conforme será abordado a seguir.

\section{OBJETO DE APRENDIZAGEM: FORMAÇÃO INICIAL E CONTINUADA DE PROFESSORES}

O nível de inserção dos dispositivos móveis nos diferentes contextos educativos faz com que espaços de capacitação docente se tornem cada vez mais necessário, em nível inicial ou continuado. Cunha (2013) salienta que a formação de professores pressupõe iniciativas instituídas ao longo da vida profissional dos mesmos, com diferentes formatos e durações, através da compreensão da formação como processo, que ocorre ao longo do tempo. Nesse sentido, a prática pedagógica é constantemente pensada e reelaborada, tendo como suporte uma epistemologia e saberes que são constantemente construídos. A partir dessa perspectiva entende-se que é essencial proporcionar aos professores a oferta de cursos de capacitação que evidenciem a importância das tecnologias digitais, bem como a reflexão sobre as possibilidades das mesmas para o processo de ensino e de aprendizagem.

Carneiro e Silveira (2014) apontam que um importante elemento facilitador do 
processo de aprendizagem são os Objetos de Aprendizagem. De acordo com Behar et al (2009b, p.67), um OA pode ser considerado "qualquer material digital, como, por exemplo, textos, animações, vídeos, imagens, aplicações, páginas web de forma isolada ou em combinação, com fins educacionais". Dessa forma, um objeto pode ser qualquer material que possui fins educativos e embasamento pedagógico. Além disso, são materiais que possam ser destinados a situações de aprendizagem tanto na modalidade à distância, quanto presencial.

Torrezzan e Behar (2013) destacam que a principal característica de um objeto consiste na possibilidade de ser reutilizável, abrangendo diferentes conteúdos, estratégias pedagógicas, por parte do professor, e estilos de aprendizagem, por parte do aluno. Por não apresentar uma estrutura linear, na sua maioria, permite ao usuário a navegação de acordo com seus interesses e percepção sobre o tema, configurando-se, assim, como uma importante ferramenta educacional.

Com base no exposto, é possível observar a importância e as possibilidades no uso desse tipo de material para a formação de professores, tanto inicial como continuada. Esse uso pode ser realizado como o conteúdo de uma Arquitetura Pedagógica, por exemplo, que possibilite a formação dos professores, introduzindo-os à essas novas perspectivas educacionais. Nessa perspectiva, a seguir é apresentada uma Arquitetura Pedagógica tendo como aspecto de conteúdo o uso de um objeto de aprendizagem denominado EduMobile.

\section{METODOLOGIA}

Esta pesquisa foi realizada em uma abordagem qualitativa e aplicando o método denominado estudo de caso. Desse modo, o estudo de caso possibilitou o desenvolvimento de uma Arquitetura Pedagógica para a M-Learning com foco na formação de professores, utilizando o objeto de aprendizagem EduMobile ${ }^{1}$.

O público-alvo foi formado por professores da rede pública atuantes da Educação Básica que estavam realizando um curso de formação continuada na Universidade Federal do Rio Grande do Sul. A coleta de dados foi realizada no ano de 2017 em um curso de extensão realizado na modalidade semipresencial, com aulas presenciais e a distância, sendo utilizado um ambiente virtual de aprendizagem. O AVA foi utilizado principalmente para a postagem de atividades e para a realização de interações em fóruns e espaço para trocas de mensagens. O curso foi ofertado com certificação de 40 horas e contou com 9 participantes. A referida AP foi aplicada e avaliada no final do curso. Para a análise dos dados os seguintes instrumentos foram utilizados: observação participante no curso e questionário no final do processo. Estes dados foram apreciados de acordo com análise de conteúdo proposto por Moraes (1999). Os dados permitiram o desenvolvimento da AP de acordo com as seguintes categorias: aspectos organizacionais, tecnológicos, metodológicos e de conteúdos.

Na próxima seção são apresentados alguns extratos coletados em questionários, atividades e registros em discussões nos fóruns realizados no ambiente virtual de aprendizagem utilizado, visando analisar a percepção dos participantes acerca do questionário que foi aplicado no final do curso.

\section{RESULTADO E DISCUSSÃO DOS DADOS}

Para responder ao objetivo da pesquisa foi, inicialmente, desenvolvida uma AP

${ }^{11}$ Disponível em: http://www.nuted.ufrgs.br/oa/edumobile/ 
com foco na formação dos professores para a utilização dos dispositivos móveis em sala de aula. Para a primeira etapa de construção da AP foi desenvolvido o objeto EduMobile: Estratégias pedagógicas para o uso da M-Learning em sala de aula.

O objeto apresenta uma estrutura com quatro módulos que abrangem os seguintes temas: (1) Dispositivos móveis: que abordam diferentes perspectivas e conceitos sobre dispositivos móveis, além de suas principais características, tipos de dispositivos e as possibilidades de uso educacional; (2) Aplicativos: que apresenta a definição de aplicativos, principalmente voltados aos dispositivos móveis, suas principais funções, categorias e seu uso na educação; (3) M-Learning: que trata da definição de aprendizagem móvel e seus principais desafios, atentando aos aspectos econômicos e de acessibilidade. (4) Estratégias pedagógicas: que elenca diferentes formas de utilização de dispositivos móveis e aplicativos na educação, além de estratégias que podem ser adotadas pelo professor de modo a promover a aprendizagem móvel em sala de aula.

Todos os módulos apresentam Materiais de Apoio, que são disponibilizados através de diferentes mídias e buscam propiciar uma abordagem abrangente dos assuntos. Também são apresentados os desafios, que se configuram como propostas e atividades relacionadas ao tema de cada módulo. Os módulos do objeto de aprendizagem podem ser acessados na ordem em que se apresentam na interface ou segundo a escolha do usuário, de modo a respeitar o raciocínio de cada sujeito.

$O$ curso foi organizado semanalmente, sendo que em cada semana foi disponibilizado um conteúdo digital para leitura, juntamente com atividades a serem desenvolvidas. $\mathrm{Na}$ avaliação foram consideradas as produções, a participação nas atividades, o envolvimento, o comprometimento e a pontualidade na entrega dos trabalhos. Na figura 1, é apresentada a AP que foi desenvolvida para o curso de extensão M-Learning e suas possibilidades no processo de ensino e aprendizagem.

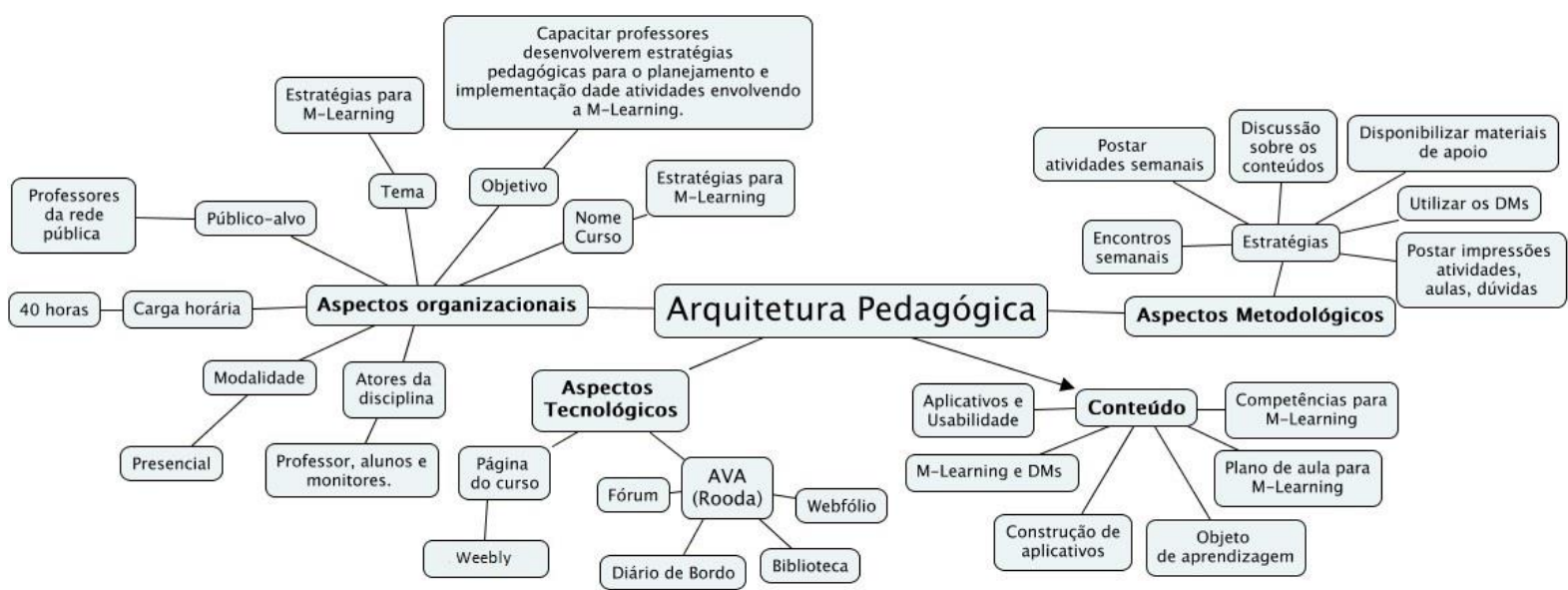

Figura 1. Arquitetura Pedagógica para um curso de formação de professores.

Fonte: As autoras (2017).

Com base na AP inicial construída e utilizada (Figura 1), o objetivo da pesquisa foi realizar uma avaliação sobre a mesma. Os participantes possuíam idades entre 26 e 56 anos, sendo atuantes em diferentes áreas de ensino, como, por exemplo, ciências biológicas, física, matemática, línguas inglesa e portuguesa. A partir de suas percepções, a seguir são avaliados os aspectos da AP aplicada.

\subsection{ASPECTOS DE CONTEÚDO}

Os aspectos de conteúdo se referem ao assunto que será abordado na AP. Assim, 
com base nesta definição, a Arquitetura proposta teve como foco o uso dos dispositivos móveis na educação. Portanto, o conteúdo da AP foi dividido em cinco temas: $M$ Learning e Dispositivos Móveis, competências para M-Learning, exemplos de aula para aprendizagem móvel, aplicativos e usabilidade e construção de aplicativos (figura 2).

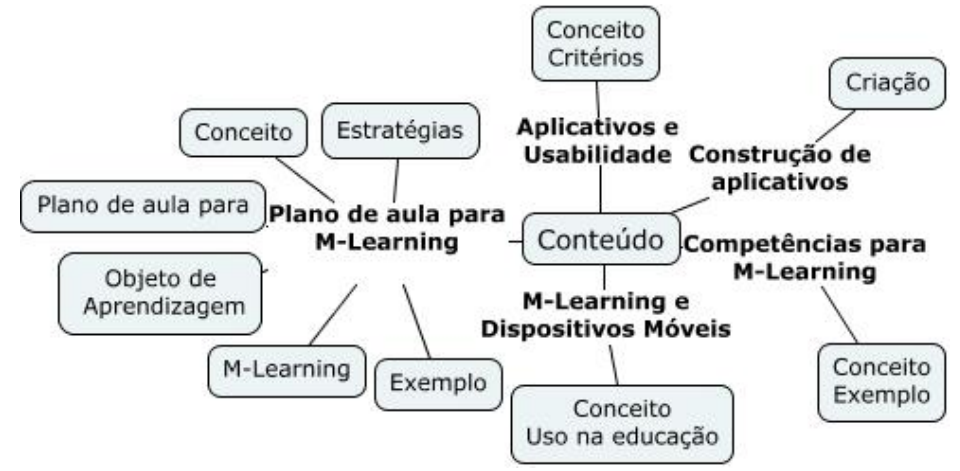

Figura 2. Aspectos de conteúdo da AP para um curso de formação de professores Fonte: As autoras (2018)

Ao longo das atividades, foram abordados os conceitos principais referentes a cada um dos temas, bem como suas relações para a educação. Entende-se que estes conteúdos devem ser apresentados com potencial para despertar o interesse, a curiosidade e a motivação no aluno, além de propiciar uma metarreflexão, ou seja, uma reflexão sobre a prática pedagógica adotada por cada professor. Portanto, considerando estes elementos, este aspecto foi analisado de acordo com as temáticas propostas para a Arquitetura Pedagógica, o que inclui o uso do OA EduMobile e a construção a partir dos conteúdos, como pode ser observado na fala dos participantes ${ }^{2}$ : "O esclarecimento é necessário para nós, professores, no sentido de que, ainda que já estejamos familiarizados com o uso de aplicativos, esta noção da utilização com o objetivo de aprendizagem, e não aleatória, é muito importante para nossa prática pedagógica" (Participante $\mathrm{M}$ ); "Além de trabalharmos com M-learning, o curso tem possibilitado a reflexão acerca dos processos de ensino e aprendizagem e nos conceitos que envolvem a avaliação" (Participante R).

Foi possível observar nos depoimentos dos participantes que os assuntos abordados foram relevantes para o desenvolvimento de atividades relacionadas com o uso dos dispositivos móveis. Observou-se, ainda, que muitos professores ainda não aprofundaram esta temática em sua formação pedagógica. Entre os principais motivos estão o fato de ser uma tecnologia relativamente recente e de muitos ambientes escolares não possuírem estrutura física para a sua aplicação.

Como destaca Martins et al (2018, p.5), simplesmente inserir as tecnologias móveis na escola "sem criar uma proposta didático-pedagógica consistente (...) não permite explorar suas potencialidades e possibilitar o desenvolvimento de práticas educacionais diferenciadas". Dessa forma, o estudo sobre o tema foi visto como fundamental para todos os participantes.

\subsection{ASPECTOS ORGANIZACIONAIS}

Os aspectos organizacionais correspondem aos elementos do planejamento de uma AP, como por exemplo, os objetivos delineados, os atores, o público-alvo, o tempo e a modalidade do curso. Na figura 3 são apresentados os elementos organizacionais que

\footnotetext{
${ }^{2}$ Visando preservar a identidade dos participantes a distinção dos mesmos foi realizada através da inicial do nome.
} 
compuseram a AP desenvolvida.

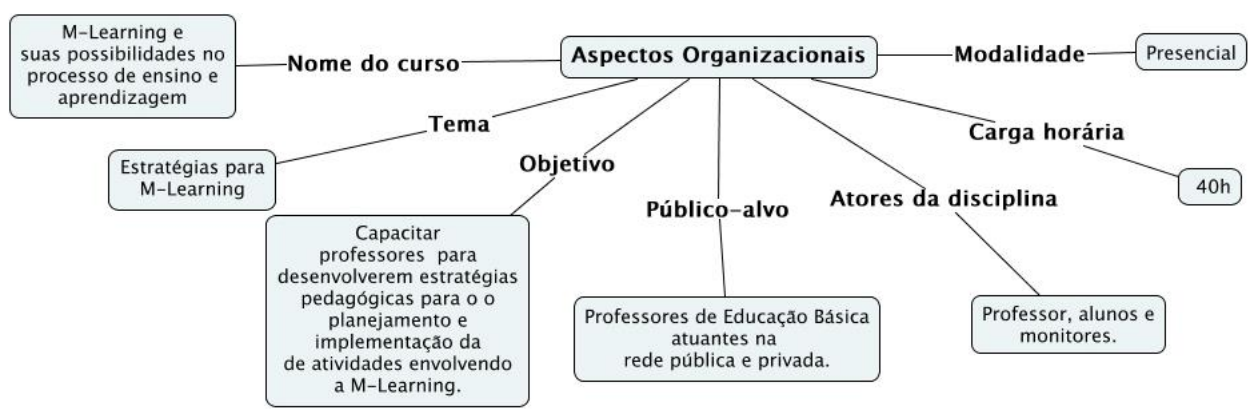

Figura 3. Aspectos organizacionais da AP para um curso de formação de professores.

Fonte: As autoras (2017).

Considerando esses elementos apresentados no Figura 3, os participantes sinalizaram que o planejamento desenvolvido propiciou uma autonomia, conforme alguns depoimentos: "Entendo que planejamento do curso foi satisfatório, como as atividades que precisam ser feitas em casa, isso nos exigiu uma competência a mais de autonomia" (Participante M); "O planejamento do curso foi satisfatório, porém, para que pudesse honrar com o que foi planejado em função das atividades precisaram ser feitas em casa, tivemos que utilizar um tempo não previsto em nosso planejamento pessoal para a dedicação ao curso. Vendo pelo lado positivo, como a colega, acabamos por exercitar um pouco mais de nossa competência de autonomia" (Participante E); "Penso que para a introdução ao assunto a troca de opiniões que fazemos faz toda a diferença para darmos continuidade por meio das atividades EAD. No entanto, como alguns colegas já disseram, o plano precisou ser adaptado a realidade que se impôs (e nada mais real que isso, não?), o que nos exigiu aplicarmos nosso poder de autonomia, que tudo tem a ver com ensino EAD. Uma oportunidade de exercitarmos essa nossa atitude" (Participante I).

Já em relação ao formato do curso, os participantes apontaram como adequado, principalmente por ser a distância, conforme a fala de um sujeito: "Achei o planejamento do curso adequado, especialmente quanto a sua estrutura compacta e objetiva. Muitas vezes verifica-se planos de aula institucionais que contemplam códigos e esquemas, não contemplando aspectos organizacionais que são essenciais para o sucesso da aula (Participante V)".

Desse modo, os participantes apontaram que os aspectos organizacionais foram satisfatórios, já que contemplou a temática proposta que está relacionada ao uso dos dispositivos móveis na educação. Pode-se observar que na formação de professores é importante considerar a falta de tempo para a realização de cursos presenciais e que a modalidade a distância pode se tornar uma excelente oportunidade de reflexão e construção do conhecimento para a formação continuada de professores, conforme será observado a seguir.

\subsection{ASPECTOS METODOLÓGICOS}

Os aspectos metodológicos remetem às estratégias de ação desenvolvidas a fim de abranger formas de comunicação, interação e avaliação dos alunos. Nesse contexto, na AP desenvolvida buscou-se promover reflexões e discussões sobre os conteúdos propostos, no qual fossem sugeridas atividades semanais e avaliou-se a participação e o envolvimento dos alunos ao longo do curso. Além disso, foram planejados três encontros presenciais, sendo dois deles com o objetivo de apresentar as ferramentas que os 
estudantes poderiam utilizar para construir um aplicativo educacional (atividade planejada para o curso), conforme Figura 4.

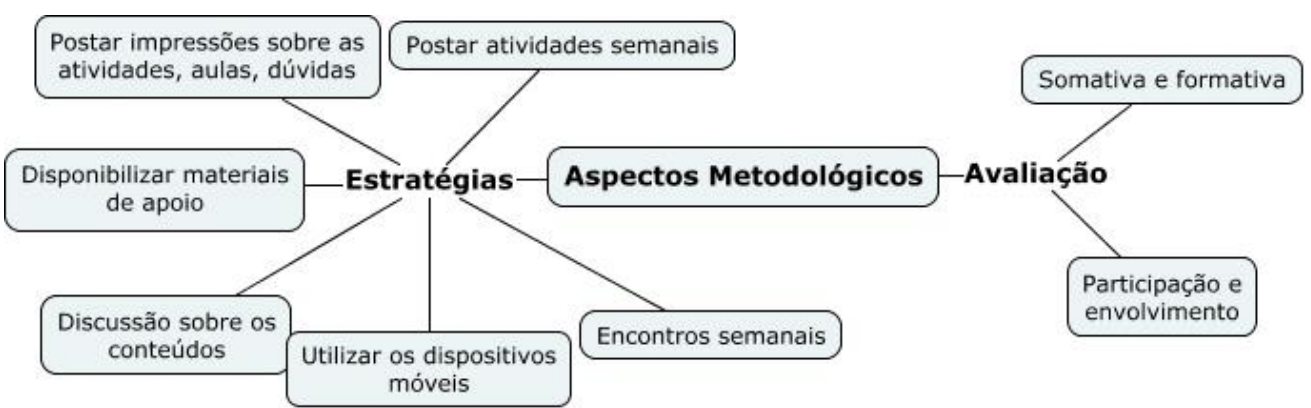

Figura 4. Aspectos metodológicos da AP para um curso de formação de professores. Fonte: As autoras (2017).

Com base nesses elementos, alguns participantes relataram: "Creio que foi disponibilizado no ROODA todos os recursos necessários para realizar a aula, contando ainda com alguns textos para aprofundar os conhecimentos" (Participante J); "Neste curso estamos vivendo isso na prática, através das atividades sugeridas, fui ficando cada vez mais curiosa sobre a imensidão de possibilidades virtuais em aplicativos que encontrei e que podem estimular especialmente a nova geração a querer aprender $e$ descobrir mais sobre qualquer assunto que lhe desperte interesse e curiosidade e que as tecnologias auxiliam muito no sentido de estimular novas descobertas" (Participante C); "Para mim foi muito positivo a contribuição do grupo, as ferramentas do ROODA, os materiais disponibilizados na biblioteca, a criação do vídeo e do app. Com certeza muito do que aprendi aqui vou levar para minha prática docente” (Participante B).

Pode-se observar que os participantes aprovaram a metodologia utilizada no curso, já que a maioria realizou as atividades propostas e apreciou a autoria de um aplicativo. Cabe salientar que este aspecto é de extrema relevância na AP, pois determina como os procedimentos adotados foram conduzidos em uma disciplina ou curso, o que, de acordo com Behar (2009) são essenciais na Arquitetura.

\subsection{ASPECTOS TECNOLÓGICOS}

Por fim, os aspectos tecnológicos para esta AP estão relacionados às formas de apresentação das atividades e dos conteúdos e nas funcionalidades utilizadas no AVA ROODA $^{3}$, plataforma desenvolvida no âmbito da Universidade Federal do Rio Grande do Sul. Para a realização do curso foram utilizadas as ferramentas que mais se adaptam à proposta, entre elas: Diário de bordo, Webfólio, Fórum, Biblioteca (Figura 5).

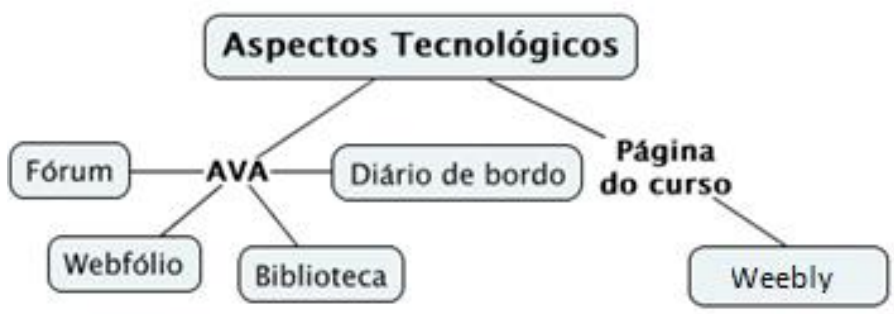

Figura 5. Aspectos tecnológicos da AP para um curso de formação de professores. Fonte: As autoras (2017).

${ }^{3}$ O ROODA está disponível em: https://ead.ufrgs.br/rooda/ 
Os participantes avaliaram este aspecto destacando, principalmente, o uso de um Ambiente Virtual de aprendizagem e sua contribuição para o desenvolvimento do curso, conforme os depoimentos a seguir: "Eu já conhecia plataformas digitais de ensino a distância e as novidades para mim, neste curso, foram: webfólio, não conhecia essa nomenclatura para postar atividades, e o diário de bordo". (Participante M); "Quanto aos recursos metodológicos e tecnológicos para a aula, penso que estão condizentes com os objetivos da aula. Destaco positivamente o uso do Diário de Bordo e do Webfólio, ferramentas que ainda não havia utilizado em outros cursos em ambientes virtuais de aprendizagem" (Participante J); "A previsão de uso do ambiente virtual (AVA) também agrega qualidade e organização ao curso. No entanto, acredito que uma possibilidade de melhoria seria melhorar a capacidade de upload do webfólio, especialmente quando se trata de um curso sobre aprendizagem a partir de desenvolvimento de aplicativos para equipamentos móveis" (Participante V).

Com estes dados é possível observar que o AVA utilizado foi importante para os participantes, principalmente a partir de questões relacionadas com interações sociais como o Webfólio (comentários) e Diário de Bordo. Entende-se, dessa forma, utilização de ferramentas de interação é primordial, já que possibilita uma maior troca de experiências e discussão sobre o tema, principalmente sobre a M-Learning.

Considerando a análise sobre a AP aplicada à formação de professores da $M$ Learning foi possível observar que esta atendeu aos objetivos propostos. Nessa perspectiva, entende-se que as estratégias utilizadas para aplicá-la são de suma importância, pois são estas que promovem dinamicidade à $\mathrm{AP}$ e comportam ajustes que poderão surgir no decorrer das atividades de ensino. Além disso, destaca-se que foram planejadas outras estratégias de ação que coladas em prática em cursos de formação futuros.

\section{CONSIDERAÇÕES FINAIS}

O objetivo da pesquisa foi propor e avaliar uma Arquitetura Pedagógica para a $M$ Learning com foco na formação de professores. Isso, pois, percebe-se que no contexto educacional existe uma crescente necessidade de inclusão dos dispositivos móveis. Paralelamente, a produção de materiais educacionais digitais, como os Objetos de Aprendizagem, tem crescido, principalmente, nos meios acadêmicos, como uma possibilidade para a apresentação de conteúdos e materiais de uma forma mais interativa e lúdica. Dessa forma, entende-se que o desenvolvimento de materiais, a exemplo do EduMobile, pode contribuir de maneira significativa, tanto para a formação de professores, quanto para material de apoio para a prática dos docentes. Com a presente investigação foi possível apresentar uma AP que pode ser usada em formações de professores para o uso dos dispositivos móveis, além de disponibilizar no repositório da Universidade um objeto de aprendizagem gratuito sobre M-Learning. Pretende-se que o EduMobile seja utilizado em qualquer curso de formação para professores, tutores e alunos sejam em cursos presenciais ou à distância.

Através da análise dos dados pode-se perceber uma contribuição da AP e do objeto na formação de professores por diferentes motivos, seja pelas reflexões acerca da ressignificação dos dispositivos móveis para seu uso na educação, ou, ainda, uma melhor compreensão do que são os materiais educacionais digitais e/ou de objetos de aprendizagem que são estudados ao longo do curso. Além disso, entende-se que será possível, futuramente, aprimorar o objeto desenvolvido, com o intuito de acrescentar novas informações e atividades relevantes à temática proposta. Também será viável 
implementar uma nova versão da AP a fim de validá-la contemplando os seus aspectos para o uso dos dispositivos móveis.

Com base nessas questões, acredita-se que é essencial que o conteúdo de uma Arquitetura Pedagógica esteja alinhado com seus aspectos metodológicos, organizacionais e tecnológicos. Nesse sentido, todos os elementos da AP devem ser contemplados para que as necessidades do curso sejam atendidas, bem como os objetivos alcançados. Além disso, a delimitação dos aspectos permite que fatores relacionados aos fatores pessoais, emocionais e sociais sejam considerados, tendo em vista as necessidades dos professores em processo de formação.

\section{REFERÊNCIAS BIBLIOGRÁFICAS}

BEHAR, P. A. Modelos Pedagógicos em Educação a Distância. In: BEHAR, P. A. (Org.) Modelos Pedagógicos em Educação a Distância. 1ed. Porto Alegre: Artmed, 2009, p. 15-32.

BEHAR, P. A.; BERNARDI, M.; SILVA, K. K. A. Arquiteturas Pedagógicas para a Educação a Distância: a construção e validação de um objeto de aprendizagem. In: RENOTE - Revista Novas Tecnologias na Educação, Porto Alegre. V. 7, No 1, Julho, 2009a.

BEHAR, P. A.; MACEDO, A. L.; SOUZA, A. P. F. C.; BERNARDI, M. Objetos de Aprendizagem para Educação a Distância. In: BEHAR, P. A. (Org.) Modelos Pedagógicos em Educação a Distância. 1ed. Porto Alegre: Artmed, 2009b, p. 66-92.

CARNEIRO, M. L.; SILVEIRA, M. Objetos de Aprendizagem como elementos facilitadores na Educação a Distância. Educar em Revista (Impresso), v. 4, p. 235-260, 2014.

CETIC.BR - Centro Regional de Estudos para o Desenvolvimento da Sociedade da Informação. Educação e Tecnologias no Brasil: um estudo de caso longitudinal sobre o uso das tecnologias de informação e comunicação em 12 escolas públicas. São Paulo: Comitê Gestor da Internet no Brasil, 2016.

CUNHA, M. I. O tema da formação de professores: trajetórias e tendências do campo na pesquisa e na ação. Educação e Pesquisa (USP. Impresso), v. 39, p. 609-625, 2013.

MARTINS, E. R.; GERALDES, W. B.; AFONSECA, U. R.; GOUVEIA, L. M. B. Tecnologias Móveis em Contexto Educativo: uma Revisão Sistemática da Literatura. In: RENOTE - Revista Novas Tecnologias Na Educação, Porto Alegre. v. 16, p. 1-10, 2018.

MORAES, Roque. Análise de conteúdo. Revista Educação, v. 22, n. 37, p. 7-32, 1999. TORREZZAN, C. A. W.; BEHAR, P. A. Competências para a Construção de Materiais Educacionais Digitais Baseados no Design Pedagógico. In: Patricia Alejandra Behar. (Org.). Competências em Educação a Distância. 1ed.Porto Alegre: Penso, 2013, v. 1, p. 237-262.

UNESCO. Diretrizes de políticas para aprendizagem móvel. Tradução de Rita Brossard. 